\title{
La Facultad de Ciencias Económicas
} y Sociales de la Universidad de los Andes y su relación con la Pequeña y Mediana Industria Merideña*

\author{
Rincón de Parra, Haydeé Cecilia**
}

\section{Resumen}

La Universidad y el sector productivo, específicamente el sector de la Pequeña y Mediana Industria, tienen mucho en común, ambos contribuyen con el desarrollo económico y social, con la generación de empleos y con el bienestar general de la población. Con el presente trabajo se tiene como propósito determinar el alcance y potencialidad de la relación la Facultad de Ciencias Económicas y Sociales de la Universidad de los Andes con la Pequeña y Mediana Industria del Estado Mérida, en lo que respecta a la prestación de servicios en materia de asesoría y asistencia técnico-administrativa y gerencial. La investigación corresponde a un estudio exploratorio-descriptivo. Se aplicó como instrumento un cuestionario con preguntas cerradas y de elección múltiple, y dos preguntas abiertas para recoger sugerencias y comentarios; a un grupo de profesores de la Facultad, y a un grupo de representantes de algunas empresas, previamente seleccionadas. Los resultados revelan que no existe una relación efectiva (estable, coherente y provechosa) entre los dos sectores estudiados, sin embargo, existe disposición tanto de los empresarios, como de los docentes para institucionalizar una relación provechosa para ambos sectores.

Palabras clave: Vinculación universidad y sector productivo, Pequeña y Mediana Industria, asistencia administrativa-gerencial.

Recibido: 01-09-28 . Aceptado: 02-07-19

Resultados del proyecto de investigación financiado por el Consejo de Desarrollo Científico, Humanístico y tecnológico (CDCHT) de la Universidad de los Andes.

** Participante del Doctorado en Ciencias Administrativas del Convenio UNESR-UNET. Magíster en Gerencia de Empresas, Mención Industrial. Licenciada en Contaduría Pública. Profesora Asociada de la Cátedra de Contabilidad de Costos, de la Facultad de Ciencias Económicas y Sociales de la Universidad de los Andes, Mérida-Venezuela. Telefax: 0274-2443269.

E-mail: haydocc@yahoo.com. 


\section{The University of Los Andes Faculty of Economic and Social Sciences and its Relationship With Small and Medium Sized Industry in Merida}

\section{Abstract}

The university and the productive sector, specifically small and medium sized industrial sector, have much in common. Both contribute to economic and social development and the generation of employment and general well-being in the population. The purpose of this paper is to determine the present level and potential of the relationship between the University of Los Andes Faculty of Economic and Social Sciences and the small and medium-sized industrial sector in Merida State in reference to services offered in the area of technical and administrative management counseling. It was a descriptive-exploratory study. A questionnaire with closed, multiple-choice and two open answer items in order to handle suggestions and comments was applied to a group of professors in the Faculty, and a group of business sector representatives previously selected. The results reveal that there is no effective (stable, coherent and beneficial) relationship between these two sectors studied, however there is interest on both parts to institutionalize a relationship between them.

Key words: Relationship university-productive sector, small and medium sized industry, administrative and management assistance.

\section{Introducción}

Los tiempos actuales, caracterizados por la globalización, la competitividad y las tecnologías de la información y la comunicación, hacen que aparezcan nuevas exigencias en materia de educación superior; de ahí que ésta tenga la necesidad de establecer nuevas políticas y estrategias que fortalezcan su rol dentro de la sociedad.

Las transformaciones de un mundo globalizado, no sólo generan cambios en la economía y los valores, sino que influyen fuertemente en el sector educativo. Se habla de la importancia que tienen los conocimientos como fuente importante de progreso y el papel que debe tener la ciencia y la tecnología en el desarrollo de las naciones, situación ésta que obliga a establecer relaciones muy estrechas entre el sistema educativo y la sociedad, y en particular, entre la universidad y el sector económico.

Al respecto Espinoza (1999) señala que la Universidad tiene una gran capacidad y potencialidad para integrarse al soporte y desarrollo económico de la sociedad. Por lo tanto no puede, ella misma, restringirse a desempeñar exclusivamente el rol de proveedora de personal altamente calificado, sino que debe tratar de extender las fronteras del conocimiento.

La necesidad de dotar a la Universidad de una proyección social más consecuente con el papel que desempeña, hace surgir con fuerza, la pertinencia de una estrecha relación (en todas sus funciones sustantivas: Docencia, Investigación y Extensión) de ésta con el mundo 
productivo, potenciando los múltiples beneficios que pueda traer consigo para ambas partes, así como para la sociedad en general. En este sentido Silvio $(2000: 119)$ señala que las universidades están estructuradas para "crear conocimientos a través de la investigación, transmitirlos a individuos necesitados y proyectarlos o "extenderlos" a la sociedad a través de servicios de asesoría en la resolución de problemas en la sociedad, a empresas y la comunidad en general" [cursivas añadidas].

En este contexto, se hace necesario estudiar si existe una relación en cuanto a prestación de servicios en materia de asesoría y asistencia técnico-administrativa y gerencial, entre la Facultad de Ciencias Económicas y Sociales (FACES) de la Universidad de los Andes (ULA) y la Pequeña y Mediana Industria (PyMI) del Estado Mérida, tomando en consideración que en la historia de la FACES-ULA, no se cuenta con estudios sobre la incidencia de la misma en el sector empresarial, específicamente sobre la Pequeña y Mediana Industria -sector fundamental para el desarrollo económico y social del Estado Mérida, de la región de los Andes y del país en general-. En consecuencia, este estudio persigue como objetivo determinar el apoyo institucional de la Facultad de Ciencias Económicas y Sociales de la Universidad de los Andes, al fomento y desarrollo de la Pequeña y Mediana Industria Merideña.

Esta investigación se justifica por lo siguiente: 1) En la FACES-ULA se imparten conocimientos sobre aspectos de gestión empresarial, pero no se conocen estudios realizados para determinar si existe una interrelación entre ésta y el sector de la Pequeña y Mediana Industria merideña, en este aspecto en particular. 2) El hecho de que se viva en una continua presión económica, competencia internacional y rápido cambio tecnológico, por un lado, y por el otro, se esté en presencia de un crecimiento cada vez más acelerado del requerimiento presupuestario de la ULA, amén de los problemas de asignación de recursos por parte del Estado hacia la Universidad, han determinado que se haga necesario que la misma busque nuevas fuentes de ingresos, a través de la interacción con el sector productivo y, de esta manera, logre proyectarse o irradiar hacia la comunidad que la circunda.

\section{Metodología de Trabajo}

El proceso metodológico utilizado en la investigación se definió como exploratorio-descriptivo, y de carácter mixto en su diseño: documental y de campo. La investigación documental consistió en un estudio de textos, documentos formales, revistas y artículos de carácter especializado, a través del cual se adquirió y enriqueció el conocimiento sobre el tema objeto de estudio, por cuanto de los mismos se obtuvieron varios aspectos relevantes referidos al proceso de la relación universidad-industria. Se hizo especial énfasis en las modalidades, las motivaciones y las barreras u obstáculos que se presentan durante el proceso de relación institucional. Igualmente, se incluyeron aspectos relevantes de lo que ha sido el proceso de relación en las economías desarrolladas, en Latinoamérica y en Venezuela.

La investigación de campo consistió en el proceso de recolección de la in- 
formación, mediante la técnica de la entrevista y el levantamiento de encuestas. Se consideraron dos tipos de poblaciones o universos:

a) 201 Pequeñas y Medianas Industrias, localizadas en los municipios Libertador, Sucre, Campo Elías y Santos Marquina del Estado Mérida, según censo practicado por la Corporación de los Andes, a las cuales se aplicó la técnica del muestreo estratificado y se determinó el tamaño de la muestra de 40 empresas a encuestar, clasificadas por Rama de Actividad Económica (RAE), de acuerdo con la Clasificación Industrial Internacional Uniforme (CIIU), de la siguiente manera: RAE 31: productos alimenticios, bebidas y tabaco (12); RAE 32: textiles, prendas de vestir e industria del cuero (10); RAE 33: industria de la madera y productos de la madera, incluidos muebles (4); RAE 34 : fabricación de papel y productos de papel, imprentas y editoriales (2); RAE 35: fabricación de sustancias químicas y de productos químicos derivados del petróleo y del carbón, de caucho y plástico (2), RAE 36: fabricación de productos minerales no metálicos, exceptuando los derivados del petróleo y del carbón (4); RAE 37: industrias metálicas básicas (1); y RAE 38: fa bricación de productos metálicos, maquinarias y equipos (5).

b) 132 profesores activos, a dedicación exclusiva y tiempo completo de la Facultad de Ciencias Económicas y Sociales, según listado de profesores emitido por la Coordinación Administrativa de la FACES, agrupados en las tres escuelas adscritas a la Facultad: Economía (15), Administración y Contaduría Pública (35), y Estadística (18); y el Instituto de
Investigaciones Económicas y Sociales (9), el Instituto de Estadística Aplicada y de Computación (8) y el Centro de Investigaciones y Desarrollo Empresarial (4). Se excluyeron los profesores que estaban gozando de año sabático y de beca, así como de permiso no remunerado; y los profesores a tiempo convencional, pues se considera que la responsabilidad de estos últimos está limitada a las horas contratadas, y por consiguiente, no tendrían tiempo disponible para una eventual participación en programas de asistencia y asesorías. Se intentó aplicar el método exhaustivo, debido a que el número de profesores (132) era relativamente pequeño; además, se consideró que los costos eran bajos. Sin embargo, se presentaron algunos inconvenientes en la aplicación de la encuesta, por lo que se logró encuestar 89 profesores (67,42\% de la población). Con el resto de profesores, se presentaron las situaciones siguientes: $2,27 \%$ estaban fuera de la ciudad de Mérida para el momento en que se hizo el levantamiento de los datos; $4,73 \%$ estaba gozando de beca y año sabático para ese momento, y 25,58\% mostró una gran apatía y desinterés en la entrevista, negándose a recibir y/o responder el cuestionario.

La recopilación de los datos se obtuvo por medio de entrevistas y encuestas aplicadas a los profesores adscritos a la Facultad y a los empresarios y/o representantes de las empresas seleccionadas en el proceso de muestreo, a través de cuestionarios. El uso de la técnica de la entrevista facilitó la labor de persuasión del entrevistado, cuando mostró resistencia a dar la información requerida; tam- 
bién sirvió para establecer la posibilidad de verificar respuestas y permitió ver la reacción de los entrevistados; así como recoger expresiones que fueron de interés para el análisis de la investigación. La información recolectada se organizó y procesó a través de procedimientos estadísticos, de manera que pudo presentarse el análisis cualitativo y cuantitativo en forma sencilla, ordenada y clara.

\section{Ontología de la Relación Universidad-Industria}

La relación de la Universidad con el sector productivo se concibe, entre otros aspectos, como:

a) Un proceso intenso, sistémico; en donde intervienen diferentes actores: los representantes del mundo productivo; los representantes de los órganos rectores de la universidad, los docentes-investigadores, estudiantes y trabajadores en general; así como representantes de otros sectores de la sociedad.

b) Un proceso dinámico, que se construye en función de un contexto económico, caracterizado por los cambios, la aparición de nuevos modelos de producción basados en el saber (conocimiento) y sus aplicaciones, así como en el tratamiento de la información. Dado que el conocimiento es considerado el motor de la productividad, hoy se habla de economías basadas en el conocimiento, es decir, basadas directamente en la producción y distribución tanto de conocimientos como de información.

c) Un fenómeno complejo, por cuanto las universidades juegan tres roles distintos en el desarrollo de nuevas capacidades industriales basadas en alta tecnología. El primer y más importante rol es el de generar nuevo conocimiento científico, tanto básico, como específico multidisciplinario (aplicado). El segundo rol es de formar el talento humano requerido para el crecimiento de los centros industriales avanzados. Por último, las universidades actúan directamente como empresas, apoyando el proceso de transferencia de sus resultados de investigación, creando una red de empresas industriales. Además, la relación universidad-industria es muy compleja porque toca un gran número de aspectos medulares del desarrollo nacional, como la calidad de los recursos humanos, la cultura, la economía, etc.

d) Una relación que subyace, por un lado, en una realidad objetiva, por cuanto depende de sus estructuras y sus procesos. En la dimensión de la estructura se identifican elementos como: 1) formalización (la formalización de la relación se mide por la existencia de una entidad legal, autonomía, modo de financiamiento, evaluación y control. 2) Sistematización. Por otro lado, toca elementos de una realidad subjetiva, dado que la relación de la Universidad-sector productivo, está orientada a producir cambios en la cultura (valores, estilos, motivaciones, intereses e iniciativas individuales, actuación, mentalidad) en los miembros de las instituciones involucradas. Las diferencias culturales pudieran bloquear e inhibir el éxito de este tipo de iniciativas integradoras.

En el caso de la Universidad, implica necesariamente, una serie de cambios organizacionales, así como la necesidad de una conciencia colectiva. Las universi- 
dades han tendido a ser ambientes cerrados, bastantes inaccesibles, y poco proclives a aceptar críticas, en especial si provienen del ambiente externo, con una conducta institucional donde prevalecen los comportamientos individuales.

e) Un fenómeno muy amplio, constituido por diversas modalidades que pueden ocurrir de manera formal -convenios o contratos formalizados generalmente por escrito- e informales -tienen lugar a través de contactos personales entre investigadores o docentes universitarios y miembros de las empresas- (Correa, s/f).

Vessuri (1982) establece que las principales formas de relación que la Universidad adopta con el sector productivo, son las siguientes: a) Tareas de investigación y desarrollo particulares, sobre la base de contratos individuales de corto plazo con la industria. b) Diseño de proyectos complejos, en donde la Universidad toma parte en la ejecución de éstos hasta la implantación final, y c) La universidad se considera un socio permanente de una organización industrial y sobre la base de acuerdos de largo plazo realiza el análisis continuo del nivel tecnológico y las condiciones de procesos manufactureros e inicia nuevas actividades, mejoras e inclusive, proyectos complejos.

Otros estudios proponen que la relación Universidad-industria, se puede presentar de tres maneras diferentes: a) Por medio de los programas docentes. Esta forma de relación recoge aquellas actividades que intentan poner en contacto a los estudiantes universitarios con el mundo productivo, como parte de sus programas de estudios. Se prepara a es- tudiantes a través de pasantías industriales y proyectos específicos. b) A través de relaciones contractuales. Estas se refieren a todos aquellos acuerdos que tienen que ver con la prestación de servicios, especialmente de la Universidad a la industria, incluyendo asesorías específicas, proyectos de investigación y desarrollo, actividades de educación continuada, servicios técnicos y auditorías tecnológicas. c) Mediante formas institucionales complejas, las cuales conllevan a una participación y relación institucional con institutos de interfase, empresas universitarias, incubadoras de empresas y parques tecnológicos (CINDA, 1990).

Como puede verse, existe una variedad de mecanismos institucionales para relacionar a la Universidad con el sector productivo, comenzando con modalidades sencillas, hasta llegar a formas superiores relacionadas con la investigación.

Es por ello, que Espinoza (1999) trata de diferenciar las modalidades de relación entre la Universidad y las empresas en tres clases, siendo éstas: "vinculación, asociación y cooperación” (p. 29), lo cual supone diversos grados de compromiso, existencia de recursos materiales y humanos, procesos gerenciales, protección de derechos, entre otros aspectos, que institucionalizan el funcionamiento de este proceso de relación, más allá de iniciativas e intereses particulares de algún miembro de la universidad.

Este mismo autor define estas modalidades de relación de la manera siguiente: La vinculación, como una relación de simple transacción, con un alto grado de autonomía de cada una de las 
partes, con una duración de corto plazo y el beneficio, en gran medida, es unilateral... Entre las situaciones de vinculación se encuentran contactos entre miembros del personal académico y representantes de la empresa, el entrenamiento de personal en áreas y actividades específicas de la empresa, la asesoría y prestación de servicios para la solución de problemas puntuales. En cuanto a la asociación, señala que ésta debe entenderse como la relación en la que se da un mayor grado de dependencia de uno de los componentes. También aclara que la asociación contempla la existencia de una estructura y recursos adecuados para tal fin, que el horizonte temporal de ésta es de corto a mediano plazo y que el propósito de la misma es la búsqueda de beneficios bilaterales. Asimismo indica que la formalidad es de mediano a alto nivel y que entre las modalidades de asociación se tienen: la subvención por empresas de proyectos de investigación -para lograr resultados aplicables a situaciones específicas de las mismas-, y la negociación de asesoramiento técnico de académicos -cuando éstos se acogen al año sabático-. Por otra parte, según el autor referido, la cooperación constituye la modalidad de relación que descansa en la alianza como estrategia de compromiso. Esta relación supone la compleja disposición de una infraestructura para tal fin. El horizonte de la cooperación en la mayoría de los casos es de largo plazo. Demanda la creación de una nueva unidad organizacional para soportar la alianza. Las organizaciones en la cooperación buscan beneficios mutuos, a través del aprendizaje y la transferencia de tecnología: conoci- miento, experticia, invenciones. Por consiguiente, busca promover el desarrollo de la innovación. Asimismo para Espinoza la cooperación conlleva a la pérdida de autonomías, en este sentido, indica que las empresas tipo "joint venture" son una forma de cooperación para desarrollar negocios, a partir de la base del conocimiento universitario.

\section{Razones, Estrategias y Obstáculos de la Relación Universidad-Industria}

La vinculación entre la Universidad y el sector productivo es un fenómeno que está adquiriendo cada vez mayor importancia en el mundo. Este fenómeno se debe principalmente a que las empresas, en busca de mejores niveles de competitividad, acuden a los centros de investigación universitarios para tener acceso a los conocimientos científicos que la revolución tecnológica demanda (Solleiro, 1990).

Considerando que uno de los principales papeles de las universidades es la diseminación del conocimiento, para el sector empresarial eso se traduce en educación y entrenamiento técnico, administrativo y gerencial, actividades éstas que son consideradas cada vez más importantes, debido al reconocimiento de la contribución que pueden hacer a la productividad y a la eficiencia (Doutriaux, 1993).

Las universidades imparten enseñanza mediante: a) Programas académicos tradicionales, para estudiantes jóvenes sin experiencia profesional, para estudiantes maduros sin educación univer- 
sitaria previa, y para profesionales medios que necesitan refrescar o actualizar sus destrezas o prepararse en un área diferente. b) Desarrollo profesional y seminarios especializados. c) Enseñanza abierta de las ciencias gerenciales y administrativas.

El conocimiento y el entrenamiento que imparten las universidades deben transferirse a las empresas mediante la inserción de profesionales, asesoría y asistencia técnica-profesional, y mediante actividades de consultoría.

En cuanto a la investigación y tecnología, las universidades deben hacer estudios científicos que las beneficien tanto a ellas como a la Pequeña y Mediana Industria. En efecto, "la excelencia en investigación parece ser un requisito indispensable para una colaboración más fructífera entre la universidad y la industria" (OECD, 1984:7). La tecnología como herramienta administrativa se ha convertido en un requisito para la productividad y eficiencia; la asesoría a las pequeñas y medianas industrias en este campo, forma parte del entrenamiento o de la asistencia profesional. Sin embargo, es probable que las empresas en las áreas intensivas en tecnología necesiten vínculos especiales con las universidades para el desarrollo ulterior, adaptación y mejoras de tecnologías específicas. Uno de los vínculos más comunes es la investigación contratada directamente entre un profesor y una pequeña firma, o convenida a través del departamento de enlace industrial de la universidad para el desarrollo, la adaptación y la mejora del aspecto técnico de un producto o de un servicio.
El conocimiento y comprensión de las mutuas ventajas que pueden obtenerse a través de la relación, constituyen aspectos de gran importancia, por cuanto estas ventajas pueden ser producto de las motivaciones de cada sector, que estimuladas y potenciadas, se convierten en factores positivos para el objetivo de vincularse.

Las motivaciones para producir la relación Universidad-Industria son muchas, entre las que se destacan: las universidades pueden ver en las empresas nuevas fuentes de recursos financieros 0 una manera de insertar a los docentes y estudiantes en el mundo real de la producción. Esto último afecta positivamente a la mejor formación profesional o de postgrado, a la vez que permite identificar nuevos proyectos de investigación. Por su lado, las empresas pueden ver a la Universidad como fuente de recursos humanos de alta calificación y de importantes proyectos $y$, por tanto, como una ventana abierta hacia la ciencia y la tecnología. Al mismo tiempo, y sobre todo para las pequeñas y medianas empresas, las universidades se constituyen en fuente importante de infraestructura de alto costo 0 de información actualizada y especializada (Aguirre, 1992).

Según este autor, una vez producida la relación, los beneficios resultantes de la misma alcanzan a la empresa, a la Universidad y a la propia comunidad académica. Para la primera, los principales beneficios son el acceso al conocimiento para permitir nuevas aplicaciones y la contratación de graduados, también el estímulo a nuevas ideas que dan lugar a innovaciones, y por tanto, a la apertura de 
nuevos mercados. Para la Universidad, un beneficio importante, además de la obtención de recursos financieros adicionales, se encuentra en la posibilidad de recibir asesoramiento en planes de investigación y formación, y la posibilidad de relacionarse directamente con la producción. Para la comunidad académica los beneficios se encuentran en las oportunidades de consultoría, y, por tanto, ingresos adicionales, mayores perspectivas de carrera y oportunidades de investigación.

Para Solleiro y López (s/t:119) "las motivaciones para la interacción de organizaciones tienden básicamente a subsanar carencias de recursos: financiamiento para apoyar nuevos proyectos de investigación, por una parte; personal científico e instalaciones de investigación, por el otro".

Del mismo modo, Espinoza (1999: 55), a partir de ideas tomadas de Azaroff, Smith y Cyr, señala que las motivaciones que inciden en el proceso de relación de la Universidad con el sector productivo son los "imperativos económicos institucionales, tales como, necesidad de diversificar la fuente de fondos universitarios debido al recorte del presupuesto para investigación, falta de capacidad propia en las empresas para conducir actividades de investigación y desarrollo y su consecuente pérdida de competitividad técnica debido al incremento de la complejidad tecnológica".

Espinoza también expresa que "entre los factores de naturaleza política e ideológica se encuentran motivaciones específicas del tipo: el crecimiento de la presión social sobre la Universidad para que demuestre su relevancia social, y la búsqueda de las empresas de una imagen y reputación pública favorable a través de la relación con la Universidad".

A pesar de los beneficios que tanto la industria como la Universidad pueden obtener a través del relacionamiento, el hecho de que ambos sectores difieran en objetivos, intereses, estructuras, participantes, usuarios, resultados finales, gestión, y en otros aspectos, trae como consecuencia, que surjan barreras que tienden a entorpecer la relación. Tales barreras han sido señaladas por más de un autor y clasificadas desde diferentes ópticas. Así, Blais (1989) clasifica dichas barreras en tres grupos: barreras estructurales (en cuanto a la naturaleza de la investigación, la confidencialidad y la administración de proyectos), barreras motivacionales (tienen que ver con la suspicacia y desconfianza mutua, el pensar que sólo lo hecho en casa tiene valor y las dudas sobre el valor de la vinculación) y barreras en los procedimientos (relacionado con la dificultad para integrar equipos de trabajo interdisciplinario en las universidades, complejidad en los contratos de transferencia tecnológica y una inadecuada mercadotecnia de servicios por parte de las universidades).

Fowler y Boyle, hablan de barreras culturales; Van Dierdonck y Debackere de barreras institucionales y operacionales; Cyr de obstáculos motivacionales y procedimentales; McMullen y Melnyk, identifican obstáculos individuales que agrupan en las siguientes categorías: experiencia, tiempo, cooperación, límites motivacionales y de acuerdos (citados por Espinoza, 1999). 
Por su parte, López, Medellín, Scanlon y Solleiro (1989), en un estudio realizado en la Universidad Nacional de México y algunas empresas de México, sobre las motivaciones y barreras en la vinculación Universidad-Industria; evidencian y sintetizan las barreras que obstaculizan la relación en los siguientes aspectos: a) condiciones estructurales (brecha cultural), b) aspectos institucionales (actitud pasiva de los industriales, desconfianza mutua, brecha cultural, falta de difusión por parte de la Universidad, inestabilidad de ésta, confidencialidad, propiedad de la tecnología, renuencia de las empresas a pagar costos totales y falta de tradición en investigación y desarrollo y c) aspectos individuales (brecha cultural, problemas de comunicación). Igualmente, Arvanitis, Pirela, Mercado y Rengifo (1989:782), en un estudio realizado, determinaron que la falta de vinculaciones entre el sector ciencia y tecnología y la industria radica, entre otros aspectos, en "la dificultad de empresarios e investigadores para hablar un lenguaje común y colaborar... y en la incapacidad de los empresarios para dirigir y gerenciar al personal de investigación y desarrollo.

Dejando de lado los factores que pudieran bloquear o inhibir la relación Universidad-Industria, es necesario considerar como inaplazable, a partir del supuesto de que el conocimiento constituye actualmente el instrumento fundamental del progreso y de la equidad social, la tarea de que ambos sectores formulen estrategias para integrarse o relacionarse. En ese sentido, según Álvarez (s/f) entre las estrategias más comunes que deben darse en la Universidad para relacionarse, se señalan: a) un cambio de orientación de la investigación de las facultades y escuelas más directamente relacionadas con la industria; b) una búsqueda más sistemática de oportunidades de utilización de los resultados de la investigación realizada en la universidad; c) oferta de capacitación para empresarios o especialistas; d) consultorías sobre temas especializados; e) acreditación académica de actividades realizadas en la empresa; f) asimilación en el currículo universitario de contenidos y procesos surgidos de la innovación en las empresas; g) conferencias y seminarios especializados a personal de las empresas; h) pasantías de estudiantes en empresas para desarrollar tesis de grado; i) visitas del personal académico a la industria; j) utilización de los laboratorios de las empresas para fines docentes y científicos; y k) apertura para la realización de contratos de investigación.

En cuanto a las estrategias utilizadas por la empresa para estrechar sus relaciones con la universidad se mencionan: a) invitar a científicos de la Universidad a través de comités de consultoría de las empresas; b) enviar a empleados y administradores a continuar sus estudios a través de convenios especiales con la universidad; c) desarrollar contratos con la universidad para apoyar investigaciones a través de donaciones; d) patrocinar cátedras sobre temas de interés para la industria; e) financiar o apoyar trabajos de tesis; f) facilitar pasantías de estudiantes; g) ofrecer oportunidades de investigación; h) organizar unidades en la com- 
pañía con la misión de establecer nexos con la universidad; e i) contribuir con equipos de laboratorio.

\section{La Universidad y la Pequeña y Mediana Industria}

Hace pocas décadas atrás se tenía la percepción de que las Pequeñas y Medianas Industrias y las universidades trabajaban en sentido contrario. Durante muchos años las PyMl consideraron que la asistencia y asesoría técnico-administrativa y gerencial provenientes de las universidades era de poco valor práctico, que la pericia académica era demasiado técnica o que estaba orientada hacia los grandes negocios.

En los últimos veinticinco años de cambio social, de reestructuración económica, de competencia internacional y de aceleración tecnológica, se ha revertido tal situación en los países desarrollados: muchas firmas pequeñas, y en particular las del área de alta tecnología, sirven a los mercados globales y son dirigidas por empresarios de trayectoria, y las universidades, ansiosas por probar su aplicabilidad social, han aumentado significativamente sus relaciones con el sector público y empresarial.

Se ha cambiado la percepción, y el valor de la asistencia y asesoría técnicoadministrativa proporcionada por las universidades está siendo reconocida. Parte del impacto de las universidades en el desarrollo económico y en la competitividad internacional resulta de sus actividades de investigación y de su contribución tecnológica. Los intercambios cada vez mayores entre las instituciones de educación superior y la PyMI de países desarro- llados, se muestra en la creciente importancia de la investigación que realiza la institución universitaria en el área empresarial: por una parte, han sido reconocidos los estudios de la PyMl como una disciplina aparte por la Escuela del Pensamiento Administrativo, y por la otra, el número de cátedras didácticas universitarias (financiadas por la PyMl y los gobiernos), revistas especializadas, conferencias anuales, seminarios y simposios, han aumentado enormemente en los últimos quince años.

Otro signo del creciente espíritu de cooperación entre las PyMl y las universidades, es el refortalecimiento de las relaciones existentes y el desarrollo de nuevos vínculos, orientados hacia la educación y el entrenamiento, asistencia profesional y la investigación, siendo la universidad quien proporciona servicios, o en el caso de algunas empresas colectivas, una participante activa.

Hoy en día, en las escuelas de Administración de los países desarrollados, los programas académicos tradicionales y los de desarrollo profesional que solían ignorar a la PyMl, ahora ofrecen cursos para empresarios, cursos de administración de Pequeñas y Medianas Industrias, de finanzas para Pequeñas y Medianas Industrias, etc. Las estadísticas sobre ofertas de cursos muestran que desde 1980 las universidades han dirigido su mirada a las Pequeñas y Medianas Industrias y le han dado un lugar dentro del programa de estudios de administración, así como en la mayoría de los planes de estudios de ingeniería, que con frecuencia incluyen uno o dos cursos sobre PyMl.

En cuanto a Latinoamérica, se encuentra en la actualidad inmersa en un 
ámbito económico que la obliga a sentar las bases de políticas que potencien el sector industrial, principalmente, el de las Pequeñas y Medianas Industrias. Para alcanzar el desarrollo, la competitividad y la equidad, hace falta la participación de instituciones y políticas pertinentes ( $R e$ tamoza,1992). La industria en Latinoamérica necesita incorporar nuevos conocimientos para poder competir en los mercados internacionales; los cambios tecnológicos y las demandas de competitividad internacional hacen necesario un mayor acercamiento entre la Universidad y el sector productivo.

La promoción de las experiencias de intercambio entre el mundo universitario y el mundo de la producción para generar beneficios privados, resulta una actividad novedosa en las universidades latinoamericanas frente a sus tradicionales funciones de docencia, investigación y extensión social, constitutivas de la cultura organizacional reformista. La creciente incorporación, a partir de los años ochenta, de actividades de relación con el mercado productivo por parte de las universidades, significa cambios sustantivos en sus relaciones con la producción. Tales transformaciones están caracterizadas por la difusión de saberes bajo la forma de acuerdos de intercambio entre partes, los cuales suponen un pago o contraprestación de recursos por los bienes y servicios producidos por la Universidad. Estas nuevas relaciones entre las instituciones académicas y el sector productivo son tratadas por algunos autores como una revisión de la clásica función de extensión como "acción extramuros" de las universidades latinoamericanas, mientras para otros constituyen la emergencia de una nueva función, diferente de la extensionista, de transferencia de conocimientos científicos y tecnológicos y prestación de servicios al mercado productivo (D'Onofrio, 1997).

Señala esta autora que el estrechamiento de las relaciones con el mundo productivo se presenta tanto a las instituciones como a los autores universitarios, no sólo como una actividad de interés académico y social, sino como una estrategia de supervivencia universitaria. Este último aspecto resulta clave para entender la situación por la cual, en el contexto latinoamericano de los años 90 , los profesores y autoridades universitarias están cada vez más dispuestos a abrazar la nueva cultura de la explotación de la investigación para el lucro comercial.

Con base en lo anterior, se puede afirmar que en las universidades latinoamericanas se han ido creando diferentes instrumentos de relación Universidad-Industria, tales como parques científicos y tecnopolos. Estos parques se han convertido en efectivas incubadoras de empresas innovadoras, "en las cuales la universidad recibe a los embriones de empresas en sus propios locales, les proporciona sus servicios de apoyo administrativo, las aconseja gracias a sus recursos tecnológicos y de gerencia, e invierte a partir de su empresa de capital de riesgo" (Emond, 1994: 160).

También en la región, algunos países han creado los llamados centros de innovación tecnológica ubicados en las universidades como instrumentos institucionales que buscan facilitar la comercialización de los resultados de la investigación de la Universidad en el medio productivo, contribuyendo a generar paque- 
tes tecnológicos transferibles. La creación de los llamados núcleos de articulaciones en empresas, también ha sido una experiencia positiva en la promoción de vínculos con la Universidad.

Aún cuando hoy día, hablar de la relación Universidad-industria en América Latina se ha convertido en un hecho común, se puede afirmar que todavía no se ha logrado una relación estable entre los dos sectores debido, fundamentalmente, a: la crisis política, económica y social que viven la mayoría de los países; la no existencia de mecanismos gubernamentales que promuevan la vinculación; un número importante de universidades no realiza investigación; en la mayoría de las universidades no existen políticas institucionales para la vinculación; el sector productivo no investiga, prefiere comprar tecnologías maduras.

No obstante, es necesario señalar que en algunas universidades como la Autónoma Nacional de México; la Católica de Chile; la Sao Paulo, Río de Janeiro y San Carlos en Brasil; la de Buenos Aires, en Argentina; y la Simón Bolívar, en Venezuela se han dado experiencias exitosas de relaciones con el sector productivo.

Indudablemente, es necesario que exista una relación entre la Universidad y la Pequeña y Mediana Industria, porque este último sector constituye el motor decisivo en el desarrollo económico de cualquier región. Esto reviste carácter especial en Venezuela, como consecuencia de la situación de dependencia que siempre ha existido en lo que se refiere a la utilización de tecnología foránea, que al ser activada en nuestro medio no sirve para dar soluciones a los problemas que afronta el país, por lo que no se logra, en con- secuencia, los resultados deseados y se crean, con mucha frecuencia, situaciones conflictivas en los sectores económicos, sociales y políticos.

La Universidad Venezolana debe, entonces, propiciar una relación amplia con la industria regional y nacional, dando lugar al desarrollo de tecnologías propias y a una aplicación más racional y sistemática de los conocimientos científicos y técnicos desarrollados en el mundo, y a las prácticas gerenciales acordes con las exigencias del medio.

En Venezuela no existe un banco de datos que permita conocer con exactitud las características de las relaciones de las universidades con el sector productivo. Cada universidad realiza de una manera muy particular, acciones orientadas al relacionamiento con la industria. Sobre este particular, Espinoza (1999: 113-163) señala que "algunas universidades, de una u otra forma, se han relacionado con empresas del sector productivo, siendo muy poco lo que se conoce de estas experiencias en cuanto a las iniciativas y estrategias de relación utilizadas para la transferencia de conocimiento, ciencia y tecnología; del impacto de la relación; y de la organización que tienen las universidades para conducir la relación". Este autor hizo un estudio explorativo en las principales universidades públicas del país (Universidad Central de Venezuela, Universidad del Zulia, Universidad de los Andes, Universidad de Carabobo, Universidad Centro Occidental Lisandro Alvarado, Universidad Simón Bolívar, y Universidad Nacional Experimental de Guayana), y a través de sus rectores, recogió las opiniones sobre el estado de la situación de la relación Universi- 
dad-sector productivo y determinó que "en casi todas las universidades existe una disposición para relacionarse con el sector productivo, la cual tiende a incrementarse en la medida que ésta tiene la necesidad de adecuar su funcionamiento a los nuevos escenarios marcados por una escasez de recursos y la presión del Estado para que estas instituciones participen activamente en la generación de ingresos adicionales".

Igualmente, Espinoza (1999:164) determinó que "casi todas las universidades disponen de una infraestructura, que en el transcurso de los últimos años se ha concebido para relacionarse con el sector productivo, como son los parques tecnológicos y las empresas universitarias". El autor concluye que "si bien hay indicios de una mayor aproximación entre los dos sectores, aún persiste cierto distanciamiento que no permite avanzar aceleradamente en la relación... Y en los casos en donde ha funcionado la relación de manera estrecha, usualmente es con la industria petrolera nacional. Muy poca experiencia se tiene con empresas de otros sectores".

De manera particular, no se da una relación estrecha entre la Universidad y la PyMl, situación que se evidencia en la literatura revisada, y en las informaciones obtenidas por el investigador en las entrevistas efectuadas directamente al personal encargado de los centros que actualmente desarrollan actividades de investigación empresarial en algunas universidades del país, tal es el caso, del Centro de Estudio para la Pequeña y Mediana Empresa de la Universidad Central de Venezuela, el Centro de Investigación y Desarrollo Empresarial (CIDE) de la Uni- versidad de los Andes, el Centro de Estudios de la Empresa (CEE) de la Universidad del Zulia, el Centro de Extensión y Asistencia Técnica a las Empresas (CEATE) de la Universidad de Carabobo, y la Coordinación de Extensión Industrial de la Universidad Nacional Experimental del Táchira.

En estos centros de investigación empresarial, la interrelación UniversidadIndustria se materializa fundamentalmente a través del dictado de cursos y seminarios con connotación teórica. Generalmente, se considera el asesoramiento y la asistencia técnico-administrativa y gerencial como una actividad secundaria que no rinde beneficios suficientes para hacerla atractiva y rentable, debido a que las Pequeñas y Medianas Empresas carecen de los recursos financieros necesarios para costearse este tipo de servicios.

\section{Alcance de la relación de la Universidad de los Andes con la PYMI}

Como resultados de la investigación, después de revisada la documentación técnica de la universidad, y analizada e interpretada la información relativa a los empresarios y a los profesores, se exponen a continuación los que por su carácter relevante revisten singular importancia, en correspondencia con los objetivos formulados en la investigación.

En los 42 años que tiene de fundada la Facultad de Ciencias Económicas y Sociales (FACES) de la Universidad de los Andes (ULA), no se ha avanzado lo deseable en cuanto a la vinculación de ésta con la PyMI, a pesar de que la Escuela de Administración y Contaduría Pú- 
blica adscrita a esta Facultad, fue creada, entre otras cosas, para satisfacer las necesidades de las empresas de la región en cuanto a gerencia empresarial se refiere. Esto se evidencia, entre otros aspectos, en lo siguiente:

El Centro de Investigación y Desarrollo Empresarial (CIDE) adscrito a la FACES, en atención a su misión de estimular, fomentar y apoyar toda actividad enmarcada en el ámbito de la investigación en las ciencias administrativas-contables, así como formular, realizar y fortalecer tareas de extensión, asesorías y docencia inherentes al desarrollo empresarial, formuló y presentó en el año 1992, ante el Consejo de la FACES, para el trámite ante el Consejo Universitario para su respectiva aprobación, un Proyecto Convenio a suscribir con Corpoindustria, que tiene por objeto organizar una mutua relación de cooperación y asesoría técnica e intercambio de información sobre actividades comunes que realizan Corpoindustria y la Universidad, las cuales son de interés y pueden arrojar beneficios para ambas instituciones. Hasta la fecha, este convenio, que fue firmado el 05 de marzo de 1993, no se ha puesto en marcha.

Entre el 13 y 30 de mayo de 1994 se celebraron en la Escuela de Administración y Contaduría Pública de la FACES de la ULA, las Primeras Jornadas de Reflexión Curricular, en donde se llegó, entre otras, a las siguientes conclusiones respecto a la interrelación de la Universidad, particularmente de la FACES con la Pequeña y Mediana Industria: a) Respecto a los problemas de gerencia en las pequeñas y medianas empresas, se evidencia que éstas, en la mayoría de los casos, no son gerenciadas por profesionales en la materia. b) Existe un desconocimiento de los problemas de la Pequeña y Mediana Empresa por parte de la FACES, por no existir los vínculos necesarios entre la institución y el sector en referencia. c) La condición de no profesionales, en lo que respecta a las personas que gerencian la Pequeña y Mediana Empresa, constituye una barrera en el necesario acercamiento entre la universidad y este sector de gran significación en la economía regional y local. Se considera que el empresario que no tenga una formación profesional, presenta una mayor resistencia a relacionar su empresa con la universidad, mostrando, por tanto, poca credibilidad y confiabilidad en el papel que desempeña esta institución. Igualmente se formularon, en la referida jornada, las siguientes recomendaciones al respecto: a) Que dentro de los programas de estudio de la FACES se incorpore el análisis de la Pequeña y Mediana Empresa. b) Que la investigación y la extensión en la Escuela de Administración y Contaduría Pública, deben contribuir con la formulación de modelos de gestión adecuados a la pequeña y mediana empresa (ULA,1994).

De la encuesta aplicada a los empresarios representantes de las pequeñas y medianas industrias, las estadísticas muestran (ver Tablas 1 y 2), que $72,5 \%$ de las empresas no reciben ningún tipo de servicio de asesoría y asistencia técnico-administrativa y gerencial, y las que lo reciben $(27,5 \%)$, lo obtienen porcentualmente de la siguiente manera: $40 \%$ de empresas privadas nacionales; $20,01 \%$ de institutos públicos; $13,33 \%$ de empresas extranjeras; otro $13,33 \%$ de la 
Universidad de los Andes y 13,33\% reciben el asesoramiento de particulares. Según la opinión de los encuestados, la asesoría que ofrece la ULA está dirigida a empresas de la rama de actividad económica 33 correspondiente (industria de la madera y productos de la madera, incluidos muebles) a través de la Facultad de Ciencias Forestales y Ambientales; y a empresas de la rama de actividad económica 35 (fabricación de sustancias químicas y de productos químicos derivados del petróleo y del carbón, de caucho y plásticos) a través de la Facultad de Ingeniería. En cuanto a la FACES, los empresarios expresaron que no reciben ningún servicio de asistencia y asesoría, y que la misma sólo les ofrece, en algunas ocasiones, cursos con orientaciones más teóricas que prácticas. (Ver Tablas 1 y 2).

Lo descrito anteriormente, refleja una situación de abandono por parte de los organismos del Estado, y de manera particular por parte de la Universidad, es- pecíficamente, de la Facultad de Ciencias Económicas y Sociales, que deberían estar comprometidos de una manera $\mathrm{u}$ otra con este sector industrial, tan importante para el desarrollo del Estado Mérida y del país en general.

Con el propósito de conocer el interés de los empresarios, respecto a un instrumento que les permita vincularse con la FACES de la ULA, y beneficiarse de éste, se incluyó en el cuestionario una pregunta relativa a la necesidad y conveniencia de la implantación de un programa de asesoría y asistencia diseñado por la FACES. Los resultados de la tabla 3 revelan que sólo $7,5 \%$ de los empresarios encuestados muestran indiferencia ante esta posibilidad, mientras que $92.5 \%$, muestran receptividad a este tipo de programa debido a que ven en él una posibilidad de mejorar su gestión empresarialgerencial, mejorar los niveles de productividad y calidad y asumir un rol más competitivo. Además, según la opinión de los

\section{Tabla 1}

\section{Utilización de los Servicios de Asesoría y/o Asistencia Técnico-Administrativa y Gerencial}

\begin{tabular}{lccc}
\hline Rama Actividad Económica & $\begin{array}{r}\text { Sí } \\
(\%)\end{array}$ & $\begin{array}{c}\text { No } \\
(\%)\end{array}$ & $\begin{array}{c}\text { No responde } \\
(\%)\end{array}$ \\
\hline 31 Alimentos, bebidas y tabacos & 7,50 & 22,50 & 0,00 \\
32 Textiles y cuero & 5,00 & 15,00 & 0,00 \\
33 Madera & 2,50 & 7,50 & 0,00 \\
34 Papel, imprentas & 2,50 & 7,50 & 0,00 \\
35 químicos, petróleo y carbón & 5,00 & 5,00 & 0,00 \\
36 Minerales no metálicos & 2,50 & 5,00 & 0,00 \\
37 Metálico básico & 0,00 & 2,50 & 0,00 \\
38 productos metálicos y maquinarias & 2,50 & 7,50 & 0,00 \\
\hline Total & 27,50 & 72,50 & 0,00 \\
\hline
\end{tabular}

Fuente: elaborado por el autor con base en información proporcionada por los empresarios. 


\section{Tabla 2}

\section{Organismo que suministra los Servicios de Asesoría y/o Asistencia Técnico-Administrativa y Gerencial}

\begin{tabular}{l|cccc|c}
\hline \multirow{2}{*}{$\begin{array}{l}\text { Rama } \\
\text { Actividad }\end{array}$} & \multicolumn{4}{|c|}{ Organismos } & \multirow{2}{*}{ Otros } \\
\cline { 2 - 5 } Económica & $\begin{array}{l}\text { Institutos } \\
\text { Públicos }\end{array}$ & $\begin{array}{c}\text { Empresas } \\
\text { Extranjeras }\end{array}$ & $\begin{array}{c}\text { Empresas } \\
\text { Nacionales }\end{array}$ & $\begin{array}{c}\text { Universidad } \\
\text { de los Andes }\end{array}$ & \\
\hline 31 Alimentos, bebidas y tabacos & 0,00 & 0,00 & 20,00 & 0,00 & 0,00 \\
32 Textiles y cuero & 6,67 & 0,00 & 0,00 & 0,00 & 6,67 \\
33 Madera & 0,00 & 0,00 & 6,67 & 6,67 & 0,00 \\
34 Papel, imprentas & 0,00 & 0,00 & 6,67 & 0,00 & 0,00 \\
35 Química, petróleo y carbón & 6,67 & 6,67 & 6,67 & 6,67 & 6,67 \\
36 Minerales no metálicos & 0,00 & 6,67 & 0,00 & 0,00 & 0,00 \\
37 metálico básico & 0,00 & 0,00 & 0,00 & 0,00 & 0,00 \\
38 productos metálicos y maquinaria & 6,67 & 0,00 & 0,00 & 0,00 & 0,00 \\
\hline Total & 20,01 & 13,33 & 40,00 & 13,33 & 13,33 \\
\hline
\end{tabular}

Fuente: elaborado por el autor con información proporcionada por los empresarios.

propios empresarios, programas de esta naturaleza se hacen necesarios, debido a que la parte industrial está desprovista de este tipo de mecanismo, que podría darle un empuje significativo al sistema productivo del Estado Mérida. También los empresarios opinan que para el desarrollo de un programa de esa naturaleza, se debe partir de un diagnóstico de la empresa que lo requiera, generando respuestas específicas y realistas para abarcar lo posible y adaptarlo a la cultura empresarial de la misma. Comunicar acertadamente la importancia de la asesoría, ya que para el empresario medio, ésta es algo extraña o novedosa, por lo tanto no es muy afecto a este tipo de relaciones (Ver Tabla 3).

De la encuesta aplicada a los profesores de la FACES, los resultados reflejan, el poco esfuerzo que se hace en extensión, pues sólo $28,09 \%$ de los encues- tados realizan actividades de extensión (Tabla 4). La extensión debe concebirse como una correspondencia permanente entre la Universidad y su ámbito externo, por lo tanto, la Universidad deberá proyectar sobre la comunidad lo que ella representa, y esto sólo lo logra, entre otros medios, a través de charlas, cursos, seminarios, asistencia, asesorías y estudios. Algunas actividades declaradas por los encuestados como de extensión son: "Tesorero de la Asociación de Profesores de la Universidad de los Andes (APULA)", "Gerente de la Empresa Promotora de Proyectos de la Universidad de los Andes (PROULA)", "Director de Relaciones Interinstitucionales de la Universidad de los Andes (ORI-ULA)", Coordinadora de la Oficina de Relaciones Interinstitucionales de la Facultad de Ciencias Económicas y Sociales (ORI-FACES)". Como puede verse, estas actividades no tienen vincu- 


\section{Tabla 3 \\ Opinión de los Empresarios Sobre la Implantación de un Programa de Asesoría y Asistencia Diseñado por la FACES}

\begin{tabular}{|c|c|c|c|c|c|}
\hline $\begin{array}{l}\text { Rama } \\
\text { Actividad } \\
\text { Económica }\end{array}$ & $\begin{array}{c}\text { Neces } \\
\text { Inmediato } \\
(\%)\end{array}$ & $\begin{array}{l}\text { ario } \\
\text { Mediato } \\
(\%)\end{array}$ & $\begin{array}{c}\text { Muy } \\
\text { Conveniente } \\
(\%)\end{array}$ & $\begin{array}{c}\text { Puede ser } \\
\text { Útil } \\
(\%)\end{array}$ & $\begin{array}{c}\text { Indiferente } \\
(\%)\end{array}$ \\
\hline 31 Alimentos, bebidas y tabacos & 2,50 & 5,00 & 2,50 & 20,00 & 0,00 \\
\hline 32 Textiles y cuero & 5,00 & 0,00 & 2,50 & 5,00 & 7,50 \\
\hline 33 Madera & 2,50 & 2,50 & 0,00 & 5,00 & 0,00 \\
\hline 34 Papel, imprentas & 2,50 & 0,00 & 0,00 & 7,50 & 0,00 \\
\hline 35 Químicos, petróleo y carbón & 2,50 & 2,50 & 0,00 & 5,00 & 0,00 \\
\hline 36 Minerales no metálicos & 0,00 & 0,00 & 2,50 & 5,00 & 0,00 \\
\hline 37 Metálica básica & 0,00 & 0,00 & 0,00 & 2,50 & 0,00 \\
\hline 38 Productos metálicos y maquinaria & 0,00 & 2,50 & 5,00 & 2,50 & 0,00 \\
\hline Total & 15,00 & 12,50 & 12,50 & 52,50 & 7,50 \\
\hline
\end{tabular}

Fuente: elaborado por el autor con información proporcionada por los empresarios.

lación con la actividad académica que desarrolla el profesor, ni constituyen en sentido estricto actividades de extensión. Asimismo, se detectó que entre las actividades propias de extensión, realizadas por los profesores, no se identifica actividad alguna relacionada con los servicios de asesoría y asistencia técnico-administrativa y gerencial al sector de la Pequeña y Mediana Industria (Ver Tabla 4).

Se consultó a los profesores sobre la necesidad y conveniencia de la implantación de un Programa de Asesoría y Asistencia Técnico-Administrativa y Gerencial diseñado por la FACES, que permita vincular a ésta, de una manera efectiva, con el sector de la Pequeña y Mediana Industria y los resultados, los cuales se muestran en la Tabla 5, reflejan que sólo $3,37 \%$ mostró indiferencia con respecto a la pregunta, pues no respondió; en tanto que $96,63 \%$ estuvo de acuerdo en cuanto a la necesidad y conveniencia de que la FACES desarrolle un programa de esa naturaleza, que permita relacionarse de una manera permanente con la industria, y así cumplir, de alguna manera, con la labor de extensión que la FACES, y por consiguiente la Universidad de los Andes, está llamada a cumplir (Ver Tabla 5).

Concuerdan las opiniones de los profesores en cuanto a que esa relación es muy positiva para: a) La ULA: porque de esta manera puede cumplir, en parte, con la función esencial de extensión. b) La Facultad: porque así podría nutrir sus cátedras con casos prácticos y reales de la problemática empresarial local. También le proporcionaría re-alimentación para conformar el perfil profesional de sus egresados, considerando las necesidades de las empresas. c) La PyMI, merideña: Por las ventajas que podría de- 


\section{Tabla 4}

Recurso docente que realiza Actividad de Extensión

\begin{tabular}{l|ccccccc|c}
\hline \multirow{2}{*}{ Respuesta } & \multicolumn{7}{|c|}{ Dependencia } & \multirow{2}{*}{ Total } \\
\cline { 2 - 7 } & $\begin{array}{c}\text { Escuela } \\
\text { Economía } \\
(\%)\end{array}$ & $\begin{array}{c}\text { Escuela Admón. } \\
\text { Contaduría Púb. }\end{array}$ & $\begin{array}{c}\text { Escuela } \\
\text { Estadística }\end{array}$ & IIES & IEAC & CIDE & (\%) \\
\hline Sí & 5,62 & 7,86 & $(\%)$ & $(\%)$ & $(\%)$ & $(\%)$ & $(\%)$ \\
No & 10,11 & 31,46 & 13,48 & 6,74 & 3,37 & 3.37 & 68,54 \\
No responde & 1,12 & 0,00 & 1,12 & 0,00 & 1,12 & 0,00 & 3,37 \\
\hline Total & 16,85 & 39,33 & 20,22 & 10,11 & 8,99 & 4,49 & 100,00 \\
\hline
\end{tabular}

Fuente: Elaborado por el autor con información proporcionada por los profesores.

IIES: Instituto de Investigaciones Económicas y Sociales.

IEAC: Instituto de Estadística Aplicada y Computación.

CIDE: Centro de Investigación y Desarrollo Empresarial.

\section{Tabla 5}

\section{Opinión de los Docentes sobre la Implantación de un Programa de Asesoría y Asistencia Técnica Diseñado por la FACES}

\begin{tabular}{l|ccccccc|c}
\hline \multirow{2}{*}{ Respuesta } & \multicolumn{7}{c|}{ Dependencia } & \multirow{2}{*}{ Total } \\
\cline { 2 - 7 } & $\begin{array}{l}\text { Escuela } \\
\text { Economía }\end{array}$ & $\begin{array}{c}\text { Escuela Admón. } \\
\text { Contaduría Púb. }\end{array}$ & $\begin{array}{c}\text { Escuela } \\
\text { Estadística }\end{array}$ & IIES & IEAC & CIDE & \\
\hline Necesario & 8,99 & 25,84 & 10,11 & 4,49 & 2,25 & 4,50 & 56,18 \\
Muy conveniente & 4,49 & 7,87 & 6,74 & 2,25 & 2,25 & 0,00 & 23,60 \\
Puede ser útil & 1,12 & 5,62 & 3,37 & 2,25 & 4,49 & 0,00 & 16,85 \\
No responde & 2,25 & 0,00 & & 1,12 & 0,00 & 0,00 & 3,37 \\
\hline Total & 16,85 & 39,33 & 20,22 & 10,11 & 8,99 & 4,50 & 100,00 \\
\hline
\end{tabular}

Fuente: Elaborado por el autor con información proporcionada por los profesores.

rivar de la asesoría técnica y profesional que promueve el proceso interactivo Universidad-Industria. d) La Región: por el efecto multiplicador que pudiera tener un programa de este tipo para el perfeccionamiento de la gestión empresarial en la Pequeña y Mediana Industria; y en el manejo y racionalización de los recursos humanos, materiales y financieros con que cuenta.

\section{Consideraciones finales}

Los datos que hemos presentado reflejan por un lado, que $72,5 \%$ de las empresas encuestadas no reciben ningún tipo de ayuda técnico-administrativa y gerencial que oriente sus esfuerzos hacia la óptima utilización de los recursos. Por otro lado, sólo $28,09 \%$ de los profesores encuestados realizan actividad de exten- 
sión, y la misma no está dirigida hacia actividades de asesoría y asistencia para el sector empresarial. Se concluye entonces, que es poca la participación de la Facultad de Ciencias Económicas y Sociales en el sector de la Pequeña y Mediana Industria del Estado Mérida, lo cual indica que la FACES no brinda un apoyo institucional -permanente-, orientado al fomento y desarrollo de este sector industrial.

Es decir, desde el punto de vista de la vinculación académica, existe un gran vacío entre la Pequeña y Mediana Industria del estado Mérida y la Facultad de Ciencias Económicas y Sociales de la Universidad de los Andes. Entre las razones o dificultades que han impedido que se dé un vínculo efectivo, a juicio de los profesores y los empresarios, se podrían mencionar las siguientes: a) Falta de confianza entre las partes, debido a que el pequeño y mediano empresario, en su mayoría, no tiene conciencia de la necesidad de formación, e incluso, tiende a despreciar como poco práctico, todo lo que tenga alguna orientación académica o connotaciones teóricas; se consideran eminentemente prácticos y son un poco reacios a invertir en tecnología, a buscar ayuda y asesorías, quieren gerenciar su empresa con un estilo todavía primitivo; por otra parte, la FACES ha estado distanciada de las necesidades del sector productivo, y ha habido una falta de difusión y promoción de las actividades que la misma realiza. b) Dificultad relacionada con la naturaleza de la actividad de investigación y docencia que debe realizar la FACES, por lo cual creemos que se deben introducir cambios curriculares y normativos). c) La empresa espera pagar bajos costos por los servicios que pueda re- cibir por parte de la FACES. d) Dificultad para integrar equipos multidisciplinarios; esta dificultad es producto de que prevalecen los intereses personales de los miembros de la comunidad de la FACES.

Por lo expuesto, se asume que uno de los mejores vínculos que se puede establecer entre el sector de la Pequeña y Mediana Industria del Estado Mérida y la Facultad de Ciencias Económicas y Sociales de la Universidad de los Andes, es mediante la implantación de un programa de asesoría y asistencia técnico- administrativa y gerencial diseñado por la FACES. Esto le permite a la misma, cumplir con uno de los objetivos de su creación, como es contribuir al desarrollo social, económico y tecnológico del Estado Mérida, de la región de los Andes y, por consiguiente, del país; y a su vez orientar a los empresarios en la solución de problemas de diversa índole y crear en ellos la expectativa de generar acciones, cuya ejecución haría posible el fortalecimiento, consolidación y crecimiento de la PyMI.

Es bueno resaltar que todo lo concerniente a la prestación de servicios de asesoría y asistencia técnico-administrativa y gerencial al sector de la PyMI, tiene que partir de la premisa de que la FACES de la ULA no es una empresa consultora. La FACES posee una cantidad de profesores de alta calidad y de características interdisciplinarias, dispuestos a colaborar con el programa de asesoría, lo que le permitiría realizar actividades que, por un lado, aporten al desarrollo de la producción o al mejoramiento de recursos humanos del sector productivo, y por el otro, que tengan un valor académico válido para la docencia y la investigación. 
Para finalizar, vale la pena señalar que en los actuales momentos en los que en la ULA se está trabajando en un proceso de cambio y transformación de la institución, y se está discutiendo el tópico "Relación de la Universidad con el Entorno", se hace oportuno proponer verdaderas políticas de vinculación con el sector externo, particularmente con el sector productivo, que conduzcan a definir estrategias de relación para la transferencia de conocimiento, ciencia y tecnología, y para la prestación de servicios de asesoría y asistencia.

\section{Bibliografía Citada}

Aguirre B., Carlos (1992), La Vinculación entre Universidad y Empresa: Un Esencial Instrumento de Desarrollo. Revista Industria y Desarrollo. № 13. Editada por el Programa de Fomento a la Pequeña y Mediana Industria de América Latina (FOPIAL), pp 9-13.

Álvarez, B. (s/f), Conocimiento y Poder: Nuevas Relaciones entre la Universidad y la Empresa. Mundo Productivo y Financiamiento. Reunión Internacional de Reflexión sobre los Nuevos Roles de la Educación Superior a Nivel Mundial: el caso de América Latina y del Caribe, futuro y escenarios deseables, UNESCO.

Arvanitis, R., Pirela, A., Mercado, A. y Rengifo, R. (1989), Empresarios y Académicos: ¿Un Matrimonio Imposible? La Capacidad de Relacionamiento y Negociación de los Empresarios con las Universidades y Centros de Investigación. ALTEC. $2^{\mathrm{a}}$ Tomo. III Seminario Latinoamericano de Gestión Tecnológica para la Modernización Industrial, Buenos Aires.
Blais, R. (1989), Condiciones para una cooperación eficaz Universidad-Industria. Revista Derecho Institucional, Año 11.

CINDA (1990), Vinculación UniversidadSector Productivo. Colección Ciencia y Tecnología № 24. Santiago de Chile.

Correa, C. (s/f), Conferencia de las Naciones Unidas sobre Comercio y Desarrollo. Universidad y Empresa en un Nuevo Escenario Competitivo La Vinculación Universidad-Empresa en la Bibliografía Latinoamericana, Naciones Unidas.

D’Onofrio, María G. (1997), Vinculación entre Universidad y Producción en los años noventa en Latinoamérica. Revista Venezolana de Gerencia, Año 3 № 5, Venezuela, La Universidad del Zulia, Vice- Rectorado Académico, pp 157168.

Doutriaux, Jérome (1993), Firmas derivadas de Universidad y otros Vínculos Universitarios con la Pequeña Empresa. Investigación y Gerencia. Revista Venezolana de Gestión. No 49, volumen X. № 4. Caracas, pp 173-184.

Emond, Alain (1994), Servicios Profesionales para las Empresas. Investigación y Gerencia. Revista Venezolana de Gestión. № 54, volumen XI. № 3. Caracas, pp 159-167.

Espinoza R., Rafael L. (1999), Naturaleza y Alcance de la Relación Universidad-Sector Productivo. Editorial de la Universidad del Zulia. Maracaibo.

López, R., Medellín, E., Scanlon, P. y Solleiro, J. (1989), La Vinculación Universidad-Industria: Motivaciones y Barreras. Ponencia presentada en el III Seminario Latinoamericano de Gestión Tecnológica para la Modernización Industrial. Tomo 3, Buenos Aires. 
OECD (1984), Industry and University: New forms of Co-operation and Communication. París.

Retamoza, V. (1992), La Educación: Base para Generación de una Posición Competitiva. Revista Industria y Desarrollo. № 13. Editada por el Programa de Fomento a la Pequeña y Mediana Industria de América Latina (FOPIAL).

Silvio, J. (2000), La Virtualización de la Universidad: ¿cómo podemos transformar la educación superior con la tecnología? IESALC / UNESCO, Caracas.

Solleiro, J. (1990), La Vinculación de la Universidad Nacional Autónoma de México con el Sector Productivo. Vinculación Universidad-Sector Productivo. Bid-Secad-Cinda. Santiago de Chile.
Solleiro y López (s/f), Conferencia de las Naciones Unidas sobre Comercio y Desarrollo. Universidad y Empresa en un Nuevo Escenario Competitivo. México: La Experiencia Reciente en la Vinculación Universidad/Empresa, Naciones Unidas.

ULA (1994), Documento sobre las Primeras Jornadas de Reflexión de la Escuela de Administración y Contaduría Pública de la Facultad de Ciencias Económicas y Sociales. Mérida.

Vessuri, H. (1982), Las Relaciones entre Universidad y Aparato Productivo. Acta Científica Venezolana. Año 1982, № 33. Asociación Venezolana para el Avance de la Ciencia. Venezuela, pp. 9-14. 